\title{
Substratos, concentrações de ácido indolbutírico e tipos de miniestacas no enraizamento de melaleuca (Melaleuca alternifolia Cheel)
}

\author{
OLIVEIRA, Y. ${ }^{1 *}$; ALCANTARA, G.B. ${ }^{1}$; GUEDES, I. ${ }^{\text {; }}$ PINTO, F. ${ }^{1}$; QUOIRIN, M. ${ }^{\text {; }}$ BIASI, L.A. ${ }^{1}$ \\ ${ }^{1}$ Laboratório de Micropropagação de Plantas, Departamento de Fitotecnia e Fitossanitarismo, Universidade Federal do \\ Paraná, Rua dos Funcionários, 1540, CEP: 80.035-050, Curitiba-Brasil *yohana@ufpr.br '²Departamento de Botânica, \\ Universidade Federal do Paraná, Caixa Postal 19031, Centro Politécnico, CEP: 81531-980, Curitiba-Brasil
}

\begin{abstract}
RESUMO: Melaleuca alternifolia tem como produto principal o óleo essencial extraído das folhas devido às propriedades antifúngicas e antibacterianas. Pouco se tem relatado sobre a propagação desta espécie, sendo a miniestaquia uma alternativa para a propagação vegetativa de clones superiores visando à implantação de campo de produção. Este trabalho teve como objetivo avaliar o efeito de diferentes substratos, concentrações de AIB, e tipo de miniestaca, no enraizamento de Melaleuca alternifolia. No primeiro experimento foram testados os substratos, areia de granulometria média, Plantmax $\mathrm{HT}^{\circledR}$, Golden-Mix ${ }^{\circledR}$ e vermiculita. No segundo experimento foram avaliadas diferentes concentrações de $\operatorname{AIB}\left(0,500,1000\right.$ e $\left.2000 \mathrm{mg} \mathrm{L}^{-1}\right)$, em dois tipos de miniestacas (apical e mediana). As miniestacas foram confeccionadas com $5 \mathrm{~cm}$ de comprimento, mantidas em casa de vegetação com nebulização intermitente, e, após 45 dias do plantio, foram avaliadas as porcentagens de miniestacas enraizadas, com calos e não responsivas, o número de raízes formadas por miniestaca e o comprimento das três maiores raízes $(\mathrm{cm})$. O substrato Golden-Mix ${ }^{\circledR}$ e as miniestacas coletadas da porção apical do ramo submetidas ao tratamento com $500 \mathrm{mg} \mathrm{L}^{-1}$ de AIB apresentaram maior porcentagem de enraizamento e melhor qualidade do sistema radicial.
\end{abstract}

Palavras-chave: Myrtaceae, auxina, óleo essencial, plantas medicinais, propagação vegetativa

ABSTRACT: Substrates, indolebutyric acid levels and types of minicuttings on the rooting of tea tree (Melaleuca alternifolia Cheel). Melaleuca alternifolia has as major product the essential oil extracted from its leaves due to its antifungal and antibacterial properties. There are scarce reports about the propagation of this species, and minicutting is an alternative for vegetative propagation of superior clones in order to establish a production field. This study aimed to evaluate the effect of different substrates, IBA levels and types of minicuttings on the rooting of Melaleuca alternifolia. In the first experiment, the following substrates were tested: medium sand, Plantmax $\mathrm{HT}^{\circledR}$, Golden-Mix ${ }^{\circledR}$ and vermiculite. In the second experiment, different IBA levels $(0,500,1000$ and $2000 \mathrm{mg} \mathrm{L}^{-1}$ ) were tested for two minicutting types (apical and medium). Minicuttings were prepared with $5 \mathrm{~cm}$ length and were kept in a greenhouse with intermittent mist; then, at 45 days after planting, we evaluated: the percentages of rooted minicuttings, with callus and non-responsive, the number of roots per minicutting and the length of the three longest roots. The substrate Golden-Mix ${ }^{\circledast}$ and the minicuttings collected from the apical part of the branch and treated with 500 $\mathrm{mg} \mathrm{L}^{-1}$ IBA presented the largest rooting percentage and the best root system quality.

Key words: Myrtaceae, auxin, essential oil, medicinal plants, vegetative propagation

\section{INTRODUÇÃO}

Melaleuca alternifolia Cheel (Myrtaceae) é uma árvore australiana nativa da região de New South Wales (Rield, 1997), conhecida mundialmente como "tea tree". Tem como produto principal o óleo essencial extraído das folhas, sendo os principais consumidores as indústrias farmacêuticas e de cosméticos, devido as propriedades antifúngicas e antibacterianas.
Embora o cultivo de Melaleuca seja economicamente lucrativo, existem poucos relatos na literatura sobre a propagação desta espécie. Lorenzi et al. (2003) indicam que a propagação das espécies $M$. armillaris, $M$. leucadendron e $M$. linariifolia é realizada por sementes.

As plantações de $M$. alternifolia são

Recebido para publicação em 19/10/2011

Aceito para publicação em 21/05/2012

Rev. Bras. PI. Med., Botucatu, v.14, n.4, p.611-616, 2012. 
normalmente estabelecidas por meio de sementes coletadas de plantas que possuem alto rendimento, entretanto, há uma variabilidade significante no rendimento e na qualidade do óleo essencial extraído dessas populações de plantas. Logo, um método de clonagem de plantas que apresentem características superiores em termos de rendimento e composição do óleo essencial, mostra-se como ferramenta importante para o sucesso da exploração da cultura em escala comercial (List et al.,1996).

A estaquia de melaleuca foi relatada por Oliveira et al. (2008), porém, os resultados de enraizamento encontrados foram pouco satisfatórios. Wendling et al. (2000) sugerem a utilização de miniestacas na clonagem de genótipos de difícil enraizamento, como por exemplo, no caso de Eucalyptus spp.

A miniestaquia proporciona incrementos em termos de enraizamento, além de favorecer o desenvolvimento de sistema radicial de melhor qualidade em termos de vigor, uniformidade e volume, o que reflete positivamente na sobrevivência e desempenho do clone no campo.

Entretanto, ainda existem poucos estudos sobre esta técnica de propagação vegetativa em espécies florestais. Santos et al. (2000) comprovaram através de estudos preliminares a viabilidade da propagação vegetativa por miniestaquia na produção de mudas de jequitibá-rosa (Cariniana estrellensis), sete-cascas (Samanea inopinata), mogno (Swietenia macrophylla) e a de cedro-rosa (Cedrela fissilis) foi estudada por Xavier et al. (2003).

Contudo, existem fatores que podem aumentar a eficiência desta técnica, como por exemplo, a utilização de reguladores vegetais, a escolha do tipo de miniestaca e do substrato empregado (Alcantara et al., 2007). Segundo Hartmann et al. (1997), aplicações exógenas de reguladores de crescimento aos propágulos vegetativos, principalmente auxinas, proporcionam maior porcentual, velocidade e qualidade de enraizamento.

O substrato utilizado para o enraizamento de estacas é um fator de grande importância na propagação vegetativa, sendo que o material ideal para a produção de mudas varia de acordo com a espécie a ser propagada e deve permitir bom suprimento de oxigênio e de água para a base da estaca e para o desenvolvimento de raízes. O substrato mais adequado deve ser inerte, poroso, com boa drenagem e capaz de manter a aeração e a umidade, permitindo o desenvolvimento do sistema radicial (Kämpf, 2000; Hartmann et al., 2002).

Diante deste cenário e visando a produção massal de clones superiores de $M$. alternifolia, este trabalho teve por objetivo avaliar o efeito de diferentes substratos, concentrações de AIB e tipos de miniestacas da espécie.

\section{MATERIALE MÉTODO}

Os experimentos foram conduzidos em casa-de-vegetação entre os meses de março a julho de 2009. As plantas matrizes foram propagadas via estaquia e mantidas em vasos com uma mistura de solo e substrato Plantmax $\mathrm{HT}^{\circledR}$ (1:1), em casa de vegetação. As miniestacas utilizadas nos experimentos foram obtidas dos ramos originados após a poda drástica destas plantas, que apresentavam, aproximadamente, dois anos de idade.

\section{Substratos}

Miniestacas medianas foram confeccionadas com $5 \mathrm{~cm}$ de comprimento, cortadas em bisel na base e reto no ápice, mantendo-se um terço das folhas na porção superior. Após o preparo, as miniestacas foram submetidas a tratamento fitossanitário com hipoclorito de sódio a $0,5 \%(\mathrm{v} / \mathrm{v})$ por $15 \mathrm{~min}$ e enxaguadas em água corrente durante $5 \mathrm{~min}$. Para o plantio foram utilizados tubetes de prolipropileno com capacidade de $53 \mathrm{~cm}^{3}$. Os substratos testados neste experimento foram areia de granulometria média, substrato comercial Plantmax $\mathrm{HT}^{\circledR}$, substrato comercial Golden-Mix ${ }^{\circledR}$ granulado Tipo 11 (a base de fibra de coco) e vermiculita de granulometria média.

Amostras dos substratos foram mantidas em estufa a $80^{\circ} \mathrm{C}$ durante $12 \mathrm{~h}$ e submetidas a análises físicas utilizando-se a metodologia de determinação rápida das propriedades físicas de substrato proposta por Fretz et al. (1979).

As miniestacas foram mantidas em casa de vegetação com nebulização intermitente e após 45 dias do plantio foram avaliadas a porcentagem de miniestacas enraizadas, com calos e não responsivas (não enraizadas e sem calos), número de raízes formadas por miniestaca e comprimento das três maiores raízes $(\mathrm{cm})$.

O delineamento experimental utilizado foi o inteiramente casualizado, com 5 repetições, 4 tratamentos e 10 miniestacas por unidade experimental. Para testar a homogeneidade das médias utilizou-se o teste de Bartlett e para a comparação de médias, os dados foram submetidos ao Teste de Tukey a $5 \%$ de probabilidade. Para a análise dos dados foi utilizado o programa estatístico SISVAR ${ }^{\circledR}$.

\section{Diferentes concentrações de AIB e tipos de miniestacas}

As miniestacas foram retiradas das porções apicais e medianas do ramo, contendo $5 \mathrm{~cm}$ de comprimento, cortadas em bisel na base e mantendose um terço das folhas na porção superior. A metodologia quanto ao tratamento fitossanitário foi a mesma do experimento anterior.

As bases das miniestacas foram imersas antes do plantio por 10 segundos em solução aquosa 
contendo AIB em diferentes concentrações $(0,500$ 1000 e $2000 \mathrm{mg} \mathrm{L}^{-1}$ ). Para o plantio foram utilizados tubetes de prolipropileno com capacidade de $53 \mathrm{~cm}^{3}$ contendo Golden-Mix ${ }^{\circledR}$ granulado Tipo 11 como substrato.

As miniestacas foram mantidas em casa de vegetação nas mesmas condições do experimento anterior, sendo avaliadas após 45 dias a porcentagem de miniestacas enraizadas, com calos, não responsivas e mortas, o número de raízes formadas por miniestaca, comprimento das três maiores raízes $(\mathrm{cm})$ e a massa fresca $(\mathrm{g})$.

O delineamento experimental utilizado foi o inteiramente casualizado, em esquema fatorial $2 \times 4$ ( 2 tipos de miniestacas $\times 4$ concentrações de AIB), com 5 repetições e 10 miniestacas por unidade experimental. A análise estatística utilizada foi a mesma do experimento anterior.

\section{RESULTADO E DISCUSSÃO}

\section{Substratos}

A maior porcentagem de enraizamento $(74,00 \%)$ foi obtida com Golden-Mix ${ }^{\circledR}$, entretanto, não houve diferença estatística com o substrato Plantmax $\mathrm{HT}^{\circledR}$. Ao se analisar as variáveis que definem a qualidade da muda formada, como o número e o comprimento das raízes, observam-se maiores valores com o substrato Golden-Mix ${ }^{\circledR}$ (Tabela 1). Como essas variáveis são de grande importância para o desenvolvimento das mudas, uma vez que permitem maior absorção de água e possíveis nutrientes adicionados ao leito de enraizamento, o substrato a base de fibra de casca de coco permitiu melhor desenvolvimento radicial de miniestacas de M. alternifolia.

De acordo com a análise das propriedades físicas dos substratos (Tabela 2), Golden-Mixâ Tipo 11 apresentou maior porosidade total $(89 \%)$ e maior retenção hídrica $(82,86 \%)$. O desenvolvimento da muda depende das propriedades físicas, químicas e biológicas do substrato que a sustenta, o que interfere diretamente na qualidade. (Lourenço et. al., 2000). Neste sentido, Carrijo et al. (2002) recomendam que o substrato ideal deve possuir, dentre outras características, porosidade acima de $85 \%$. Além disso, estes autores destacam as boas características da fibra de coco em relação a não reação com outros nutrientes da adubação, a longa durabilidade das características físicas, possibilidade de esterilização, abundância da matéria prima e o baixo custo.

O enraizamento foi favorecido com a utilização do Golden-Mix ${ }^{\circledR}$ (Tabela 1), provavelmente por ser o ambiente que melhor propiciou a retenção hídrica. A grande afinidade da Melaleuca alternifolia por ambientes úmidos deve-se ao fato da espécie ser nativa de regiões pantanosas ou próximas a rios (Rield, 1997).

Analisando a variável porcentagem de calos (Tabela 1), nota-se que o substrato areia foi o que propiciou a maior formação (30\%), podendo ser um indicativo de enraizamento futuro. As células desse tecido indiferenciado podem vir a se diferenciar e formar primórdios radiciais. No entanto, segundo Zuffellato-Ribas \& Rodrigues (2001), a formação de calos e de raízes adventícias são independentes.

Para a variável não responsiva, ou seja, miniestacas que não enraizaram e não formaram calo, o maior valor encontrado se deu com o substrato vermiculita (34\%) (Tabela 1).

\section{Diferentes concentrações de AIB e tipos de miniestacas \\ Para a variável porcentagem de} enraizamento, as miniestacas apicais apresentaram resultados superiores em relação às medianas em todas as concentrações testadas (Tabela 3). Esses resultados corroboram com Hartmann et al. (2002), que destacam que, de modo geral, as estacas da

TABELA 1. Porcentagem de enraizamento, número de raízes formadas por miniestaca,comprimento das três maiores raízes, porcentagem de miniestacas com calos e não responsivas de Melaleuca alternifolia, com diferentes substratos.

\begin{tabular}{cccccc}
\hline Substratos & $\begin{array}{c}\text { Enraizamento } \\
(\%)\end{array}$ & $\mathrm{N}^{\circ}$ raízes & $\begin{array}{c}\text { Comprimento } \\
\text { raízes }(\mathrm{cm})\end{array}$ & Calos $(\%)$ & $\begin{array}{c}\text { Não responsivas } \\
(\%)\end{array}$ \\
\hline Vermiculita & $18,00 \mathrm{c}$ & $1,50 \mathrm{~d}$ & $0,20 \mathrm{c}$ & $8,00 \mathrm{c}$ & $74,00 \mathrm{a}$ \\
Areia & $36,00 \mathrm{~b}$ & $3,20 \mathrm{c}$ & $0,90 \mathrm{bc}$ & $30,00 \mathrm{a}$ & $34,00 \mathrm{c}$ \\
${\text { Plantmax } \mathrm{HT}^{\circledR}}^{\circledR}$ & $70,00 \mathrm{a}$ & $7,60 \mathrm{~b}$ & $1,90 \mathrm{~b}$ & $16,20 \mathrm{~b}$ & $13,80 \mathrm{c}$ \\
Golden-Mix $^{\circledR}$ & $74,00 \mathrm{a}$ & $9,10 \mathrm{a}$ & $4,80 \mathrm{a}$ & $14,00 \mathrm{bc}$ & $12,00 \mathrm{c}$ \\
\hline $\mathrm{CV}(\%)$ & 18,47 & 17,60 & 40,69 & 44,36 & 30,73 \\
\hline
\end{tabular}

Médias seguidas da mesma letra na vertical, para tipo de substrato não diferem significativamente pelo teste de Tukey a $5 \%$ de probabilidade. 
TABELA 2. Determinação das propriedades físicas dos substratos. Curitiba - PR, 2009.

\begin{tabular}{|c|c|c|c|c|}
\hline Substratos & $\begin{array}{c}\text { Densidade seca } \\
\left(\mathrm{g} \mathrm{L}^{-1}\right)\end{array}$ & $\begin{array}{l}\text { Porosidade } \\
\text { total }(\%)\end{array}$ & $\begin{array}{l}\text { Retenção hídrica } \\
(\%)\end{array}$ & $\begin{array}{l}\text { Espaço de aeração na } \\
\text { capacidade recipiente }(\%)\end{array}$ \\
\hline Vermiculita & 145,83 & 66,75 & 41,61 & 25,14 \\
\hline Areia & 1764,85 & 34,88 & 29,45 & 5,43 \\
\hline Plantmax $\mathrm{HT}^{\circledR}$ & 396,3 & 66,57 & 47,67 & 18,9 \\
\hline Golden-Mix $^{\circledR}$ & 125,47 & 89,00 & 82,86 & 6,14 \\
\hline
\end{tabular}

Densidade seca $\left(\mathrm{g} \mathrm{L}^{-1}\right)=$ densidade em g L-1; Porosidade total $(\%)=$ espaço poroso total em \%; Retenção hídrica $(\%)=$ água retida na capacidade de campo (\%); Espaço de aeração $(\%)=$ espaço de ar na capacidade recipiente

posição apical de espécies lenhosas apresentam maiores níveis de enraizamento, devido à intensa atividade meristemática.

Estacas apicais apresentam nível mais elevado de auxina endógena que estacas retiradas de porções basais do ramo, pois essas substâncias se encontram principalmente nos órgãos em crescimento ativo, tais como regiões meristemáticas e, portanto, muitas vezes essas auxinas são suficientes para estimular o enraizamento de estacas apicais, dispensando o uso exógeno (Zuffellato-Ribas \& Rodrigues, 2001). Como no presente trabalho a aplicação de AIB possibilitou maior enraizamento e um sistema radicial de melhor qualidade, o que pode ser evidenciado com as variáveis número de raízes, comprimento e massa fresca, o uso de auxina exógena representa fator positivo ao enraizamento das miniestacas apicais.

A concentração de $500 \mathrm{mg} \mathrm{L}^{-1}$ foi a que proporcionou maior número de raízes e maior massa fresca, sendo que para o comprimento não diferiu da concentração de $1000 \mathrm{mg} \mathrm{L}^{-1}$ (Tabela 3). Oliveira et al. (2008), testaram diferentes tamanhos $(10,15$ e 20 $\mathrm{cm}$ ) de estacas de melaleuca, tratadas com 1500 $\mathrm{mg} \mathrm{L}^{-1}$ de AIB, e encontraram maior porcentagem de enraizamento $(41,25 \%)$ em estacas de $10 \mathrm{~cm}$ de comprimento, sendo, portanto inferior as miniestacas apicais tratadas com $500 \mathrm{mg} \mathrm{L}^{-1}$ do presente trabalho $(68,00 \%)$. Este aumento na porcentagem de enraizamento, mesmo com o uso de menor concentração de AIB, provavelmente tenha ocorrido devido ao nível mais elevado de auxina endógena presente nas miniestacas apicais. Além disso, a utilização de miniestacas, no lugar de estacas, pode ter proporcionado incremento no enraizamento e ter possibilitado a formação de sistema radicial de melhor qualidade (Alcantara et al., 2007). No entanto, porcentagem superior de enraizamento $(82,5 \%)$ foi encontrado com a aplicação de $1500 \mathrm{mg} \mathrm{L}^{-1}$ de AIB, em estudo com miniestacas de $M$. alternifolia de 6 $\mathrm{cm}$ de comprimento, no qual foram testadas diferentes concentrações de AIB $\left(0,500,1000\right.$ e $\left.1500 \mathrm{mg} \mathrm{L}^{-1}\right)$ (Costa et al., 2007).

As miniestacas apicais que receberam 2000 $\mathrm{mg} \mathrm{L}^{-1}$ de AIB apresentaram maiores médias para as variáveis número de raízes, comprimento e massa fresca, diferindo estatisticamente das demais concentrações testadas (Tabela 3). Entretanto, essa

TABELA 3. Porcentagem de enraizamento, número de raízes formadas por miniestaca, comprimento das três maiores raízes de miniestacas apicais e medianas de Melaleuca alternifolia, submetidas a diferentes concentrações de AIB (mg L-1).

\begin{tabular}{|c|c|c|c|c|c|c|c|c|}
\hline \multirow[t]{2}{*}{ AIB } & \multicolumn{2}{|c|}{ Enraizadas (\%) } & \multicolumn{2}{|c|}{ Número de raízes } & \multicolumn{2}{|c|}{$\begin{array}{l}\text { Comprimento das três } \\
\text { maiores raízes }(\mathrm{cm})\end{array}$} & \multicolumn{2}{|c|}{ Massa fresca $(\mathrm{g})$} \\
\hline & Apical & Mediana & Apical & Mediana & Apical & Mediana & Apical & Mediana \\
\hline 0 & 54,40 b $A$ & 30,00 b $\quad B$ & 3,59 C $B$ & 4,23 a $A$ & $5,74 \quad c \quad B$ & 6,68 b $A$ & 0,09 d $B$ & 0,12 a $A$ \\
\hline 500 & 68,00 a $A$ & 48,00 a $B$ & 4,84 b $A$ & 2,25 b $\quad B$ & 8,41 b $A$ & 5,84 b $\quad B$ & 0,17 b $\quad A$ & 0,11 a $B$ \\
\hline 1000 & 62,00 a $A$ & 24,20 b $B$ & 3,21 C $A$ & 2,49 b $B$ & 8,99 b A & 7,95 a $B$ & $0,13 \subset A$ & 0,11 a $A$ \\
\hline 2000 & 39,80 c $A$ & 4,40 b $B$ & 5,95 a $A$ & 0,40 c $B$ & 10,47 a $A$ & 4,70 C $B$ & 0,24 a $A$ & 0,05 b $B$ \\
\hline CV (\%) & \multicolumn{2}{|c|}{73,1} & \multicolumn{2}{|c|}{2,05} & 7,5 & & \multicolumn{2}{|c|}{8,38} \\
\hline
\end{tabular}

Médias seguidas da mesma letra minúscula na vertical, para concentração de AIB, e médias seguidas da mesma letra maiúscula na horizontal, para tipo de miniestacas, não diferem significativamente pelo teste de Tukey a $5 \%$ de probabilidade

Rev. Bras. PI. Med., Botucatu, v.14, n.4, p.611-616, 2012. 
concentração proporcionou a menor taxa de enraizamento para ambos os tipos de miniestacas. O aumento da dose de auxina exógena aplicada em estacas provoca efeito estimulador de raízes até um valor máximo, a partir do qual qualquer acréscimo de auxina tem efeito inibitório (Fachinello et al., 2005). O efeito fitotóxico de certas doses de auxina está relacionado com o fato de as estacas possuírem certa quantidade endógena de hormônios, promotores ou inibidores de enraizamento e o fornecimento exógeno de auxina pode promover alteração hormonal, favorecendo ou não o enraizamento (Hartmann et al., 2002). O AIB tem sido eficiente na formação de mudas de diferentes espécies por estaquia, porém apresenta resultados distintos dependendo da concentração e dos cultivares empregado. As auxinas podem causar efeitos fitotóxicos se aplicadas em concentrações excessivas (Costa Júnior et al., 2003).

A testemunha e a concentração de $500 \mathrm{mg}$ $\mathrm{L}^{-1}$ de AIB favoreceram a formação de calos, sendo as miniestacas apicais superiores em relação às medianas. As miniestacas medianas apresentaram maiores porcentagens de não responsivas que as apicais, com exceção da concentração de 2000 mg $\mathrm{L}^{-1}$, onde não houve diferenças entre os tipos de miniestacas (Tabela 4).

A mortalidade foi inexistente na testemunha e na menor concentração de AIB, para os dois tipos de miniestacas testadas. De forma geral, houve baixa taxa de mortalidade, com exceção das miniestacas medianas tratadas com a maior concentração de AIB (Tabela 4).

Diante dos resultados obtidos, conclui-se que o enraizamento de miniestacas de Melaleuca alternifolia é favorecido com o substrato GoldenMixâ Tipo 11. Miniestacas coletadas da porção apical do ramo, submetidas ao tratamento com 500 $\mathrm{mg} \mathrm{L}^{-1}$ de AIB, proporcionam maior porcentagem de enraizamento e melhor qualidade do sistema radicial.

TABELA 4. Porcentagem de formação de calos, não responsivas e mortalidade de miniestacas apicais e medianas de Melaleuca alternifolia, submetidas a diferentes concentrações de AIB.

\begin{tabular}{|c|c|c|c|c|c|c|c|c|c|c|c|c|c|c|c|}
\hline \multirow{3}{*}{$\begin{array}{c}\text { AIB } \\
\frac{\left(\mathrm{mg} \mathrm{L}^{-1}\right)}{0}\end{array}$} & \multicolumn{5}{|c|}{ Calo (\%) } & \multicolumn{5}{|c|}{ Não responsivas (\%) } & \multicolumn{5}{|c|}{ Mortalidade (\%) } \\
\hline & \multicolumn{2}{|c|}{ Apical } & \multicolumn{3}{|c|}{ Mediana } & \multicolumn{3}{|c|}{ Apical } & \multicolumn{2}{|c|}{ Mediana } & \multicolumn{2}{|c|}{ Apical } & \multicolumn{3}{|c|}{ Mediana } \\
\hline & 20,00 & a $A$ & 14,00 & a & $\mathrm{B}$ & 25,60 & $b$ & & 56,00 & b A & 0,00 & b $A$ & 0,00 & $b$ & A \\
\hline 500 & 22,00 & a $A$ & 14,40 & a & B & 10,00 & c & $B$ & 37,60 & C $\mathrm{A}$ & 0,00 & b $A$ & 0,00 & $b$ & $A$ \\
\hline 1000 & 8,20 & $b \quad B$ & 1,60 & $\mathrm{~b}$ & & 19,80 & bc & $\mathrm{B}$ & 68,20 & a $\mathrm{A}$ & 10,00 & a $\mathrm{A}$ & 6,00 & $b$ & A \\
\hline 2000 & 6,40 & b $A$ & 4,40 & $b$ & A & 41,80 & $a$ & $A$ & 35,20 & C $A$ & 12,00 & a $B$ & 56,00 & a & $A$ \\
\hline CV (\%) & \multicolumn{5}{|c|}{22,61} & \multicolumn{5}{|c|}{17,17} & \multicolumn{5}{|c|}{51,29} \\
\hline
\end{tabular}

Médias seguidas da mesma letra minúscula na vertical, para concentração de AIB, e médias seguidas da mesma letra maiúscula na horizontal, para tipo de miniestacas, não diferem significativamente pelo teste de Tukey a $5 \%$ de probabilidade

\section{REFERÊNCIA}

ALCANTARA, G.B. et al. Efeito da idade da muda e da estação do ano no enraizamento de miniestacas de Pinus taeda L. Revista Árvore, v.31, n.3, p.399-404, 2007. CARRIJO, O.A.; LIZ, R.S.; MAKISHIMA, N. Fibra de casca de coco verde como substrato agrícola. Horticultura Brasileira, v.20, n.4, p.533-5, 2002.

COSTA, A.G. et al. Diferentes concentrações de ácido indolbutirico no enraizamento de estacas de melaleuca. In: CONGRESSO BRASILEIRO DE OLERICULTURA, 47., 2007, Porto Seguro. Anais... Porto Seguro: Horticultura Brasileira, supl., 2007. p.39.

COSTA JÚNIOR, W.H.; SCARPARE FILHO, J.A.; BASTOS, D.C. Estiolamento da planta matriz e uso de ácido indolbutírico no enraizamento de estacas de goiabeiras. Revista Brasileira de Fruticultura, v.25, n.2, p.301-4, 2003. FACHINELLO, J.C.; HOFFMANN, A.; NACHTIGAL, J.C.
Propagação de plantas frutíferas. Brasília: Embrapa, 2005. 221p.

FRETZ, T.A.; READ, P.E.; PEELE, M.C. Plant propagation laboratory manual. Minneapolis: Burgess Publishiny Company, 1979. 317p.

HARTMANN, H.T. et al. Plant propagation: principles and practices. 6.ed. New Jersey: Prentice-Hall, 1997. 770p. HARTMANN, H.T. et al. Plant propagation: principles and practices. 7.ed. New Jersey: Prentice Hall, 2002. 880p. KÄMPF, A.N. Substrato. Produção comercial de plantas ornamentais. Guaíba: Agropecuária, 2000. p.45-73.

LIST, S.E. et al. A micropropagation protocol for Melaleuca alternifolia (tea tree). Australian Journal of Experimental Agriculture, v.6, n.36, p.755-60, 1996.

LORENÇO, R.S. et al. Influência do substrato no desenvolvimento de mudas de erva-mate (I/ex paraguariensis St. Hill.). Perspectiva, v.24, n.88, p.81-9, 2000. 
LORENZI, H. et al. Árvores exóticas no Brasil: madeireiras ornamentais e aromáticas. Nova Odessa, São Paulo: Plantarum, 2003. 173p.

OLIVEIRA, Y. et al. Comprimento das estacas no enraizamento de melaleuca. Scientia Agraria, v.9, n.3, p.415-8, 2008.

RIELD, R.W. Practical methods for using tea tree oil. Agro-Food Industry Hi-Tech, v.8, p.34-6, 1997.

SANTOS, G. A. et al. Enraizamento de miniestacas de Jequitibá rosa, Sete cascas e Mogno. Resultados preliminares. In: SIMPÓSIO DE INICIAÇÃO CIENTÍFICA
DA UFV, 2000, Viçosa. Anais... Viçosa: Universidade Federal de Viçosa, 2000. p.63.

XAVIER. A. et al. Propagação vegetativa de cedro-rosa por miniestaquia. Revista Árvore, v.27, n.2, p.13943, 2003.

ZUFFELLATO-RIBAS, K.C.; RODRIGUES, J.D. Estaquia: uma abordagem dos principais aspectos fisiológicos. Curitiba (K.C. Zuffellato-Ribas), 2001. 39p.

WENDLING, I. et al. Propagação clonal de híbridos de Eucalyptus spp. por miniestaquia. Revista Árvore, v.24, n.2, p.181-6, 2000. 